\title{
Practice-induced and sequential modulations of the Simon effect
}

\author{
Eric Soetens, Kathleen Maetens, and Peter Zeischka \\ University of Brussels, Brussels, Belgium
}

\begin{abstract}
People react more quickly and more accurately to stimuli presented in locations corresponding to the response, as compared with noncorresponding locations, even when stimulus location is irrelevant (Simon effect [SE]). The explanation that SEs are caused by the automatic priming of a corresponding response has been questioned, because of the many exceptions to the effect. We replicated practice-induced and sequential modulations of the SE in two experiments - first, by training participants with blocks of location-relevant stimuli, and second, by mixing location-relevant and location-irrelevant trials. The decrease of the SE with incompatible training was relatively permanent in the blocked experiment, whereas the effect was temporary in the mixed experiment. The difference was caused by a more permanent reversal of the SE after incongruent trials, showing that sequential modulations depend on long-term practice effects. We suggest that there is a formation of a contralateral longterm memory stimulus-response link in blocked conditions and that short-term and long-term memory links are primed by preceding events.
\end{abstract}

When participants have to respond with a left or a right response in a two-choice reaction time (RT) task, reactions are faster to an ipsilateral than to a contralateral stimulus. This effect holds even when the location of the stimulus is not relevant for the task, such as when participants have to react to the color of the stimulus. In the latter case, the difference in RT between location-corresponding trials and location-noncorresponding trials is called the Simon effect (SE; Simon, 1990; Simon \& Rudell, 1967).

The SE can change and even reverse if people practice first with a location-relevant task or when a Simon task is mixed with location-relevant trials. In serial tasks, the SE can also disappear or reverse when the preceding trial in a sequence is incongruent. Although several theories have been advanced to explain one or the other of these modulations, an adequate model explaining the interactions that occur between these modulatory effects is still missing (but see Iani, Rubichi, Gherri, \& Nicoletti, 2009, for a suggestion). In the present study, we demonstrate the mutual dependency of both modulations and propose an explanation for the pattern of results.

Several mechanisms and models have been proposed to explain the SE (for an overview, see Proctor \& Vu, 2002). The model that has the most support in the literature is the alternative-route model, where it is suggested that the effect is caused by the activation of a spatial code generated by the irrelevant spatial stimulus attribute (e.g., Kornblum, Hasbroucq, \& Osman, 1990; Kornblum \&
Stevens, 2002). According to this model, when a stimulus dimension overlaps with a response dimension, features of the same stimulus can be processed along two different routes, a controlled route and an automatic responsepriming route (e.g., Hommel \& Prinz, 1997; Notebaert, Soetens, \& Melis, 2001). The controlled route follows the intentional translation of a stimulus attribute into response activation. The response-priming route is triggered by the stimulus dimension overlapping with the response dimension, automatically activating the corresponding response. In the case of the SE, stimulus and response sets have a common spatial dimension, so that the irrelevant location of the stimulus automatically triggers a corresponding response. The relevant stimulus attribute is nonspatial, such as color or shape.

The two routes have been differentiated in terms of underlying memory processes. The intentional translation process is based on task-defined short-term associations, whereas automatic response priming is based on long-term associations (Barber \& O'Leary, 1997; Zorzi \& Umiltà, 1995). Zorzi and Umiltà developed a connectionist model to explain a wide variety of results in the Simon literature. However, the model has difficulties accommodating the reversal of the SE in some conditions. For example, the SE disappears or even reverses when participants are presented with a mixture of Simon trials and incompatible location-relevant trials (Marble \& Proctor, 2000; Proctor \& Lu, 1999; Proctor, Marble, \& Vu, 2000). In the follow-

E.Soetens, esoetens@vub.ac.be 
ing, we will refer to this type of condition as the mixed condition. Note that, from here on, we will use the terms compatible and incompatible whenever stimulus location is relevant for the task and congruent and incongruent when location is irrelevant. Also, when incompatible location-relevant trials are presented in separate blocks, prior to the Simon task, a similar modulation of the effect occurs (Iani et al., 2009; Proctor \& Lu, 1999; Tagliabue, Zorzi, \& Umiltà, 2002; Tagliabue, Zorzi, Umiltà, \& Bassignani, 2000; Vu, Proctor, \& Urcuioli, 2003). We will refer to these as the blocked condition. To meet these new findings, Tagliabue et al. (2000) extended the model of Zorzi and Umiltà. They attributed the switch to a reversed SE to active short-term memory (STM) links between stimulus position nodes and contralateral response position nodes, based on task instructions in the locationrelevant trials. These short-term associations between stimulus and response locations affect performance even when the original task is no longer in effect, so that, in a subsequent Simon task, the SE is reversed.

The claim that the Simon reversal effect is caused by STM links is theoretically based on the development of links according to the proposed connectionist model (Tagliabue et al., 2000). The data themselves rather point to the development of a long-term memory (LTM) link. First, although not explicitly stated, the explanations concerning the reversal of the SE seemed to suggest that memory links need time to develop in memory before a reversal can take place. Although some studies have shown a reversal over a very short training session of 72 trials (Tagliabue et al., 2000), a minimum number of trials seems to be necessary. Tagliabue et al. (2000) already suggested that memory traces could be consolidated. Consolidation is a process that is typically linked not to STM but, rather, to LTM. Second, in that same study, Simon reversal effects were found $24 \mathrm{~h}$ after training with an incompatible task, and the effects even seemed to strengthen over a period of 7 days. Vu et al. (2003) replicated the long-lasting reversal after 1 week but were unable to reproduce the increase of the effect. It is strange that the authors ascribed these changes to so-called "long-lasting STM links," whereas elsewhere in the literature, such effects are ascribed to LTM. It is not clear what the difference is between what the authors call "long-lasting STM links" and LTM links. A somewhat adapted explanation in terms of hippocampal versus neocortical memory systems was provided in a later study (Tagliabue et al., 2002). We will consider this model in more detail in the General Discussion section.

Although the difference in persistence of the reversal effect in blocked and mixed conditions has not been investigated directly, some studies seem to indicate that the effect is more permanent in nature in the blocked condition. Tagliabue et al. (2000) found an increase of the reversed SE when the delay between the (in)compatible location-relevant task and the Simon task increased, suggesting that a memory consolidation process is responsible for these long-term effects. Moreover, Proctor and $\mathrm{Lu}$ (1999) showed that, in a blocked condition, the reversed SE decays with practice but remains significant, just as it does with a normal SE. These findings rather seem to point to modulations of LTM links. No such consolidation or practice effects have been examined in mixed conditions. It may well be that the memory links formed in the blocked conditions are different from those in the mixed conditions. In their overview of the literature, Proctor and $\mathrm{Vu}$ (2002) pointed out that there is little doubt that task sets play a significant role in SEs. In the blocked condition, participants have the opportunity to consolidate one specific mapping rule during the location-relevant blocks, whereas in the mixed condition, the mapping rule changes from trial to trial, so that consolidation may be prevented. A first important aim of the present study was to demonstrate that the reversal in the blocked condition is more permanent in nature and, therefore, caused by a new contralateral LTM link, in contrast to the reversal effect obtained in the mixed condition.

Besides the modulatory influence of location-relevant practice, SEs can be eliminated (Notebaert \& Soetens, 2003; Notebaert et al., 2001) and even reversed when the preceding trial in a serial task is incongruent (e.g., Mordkoff, 1998; Stürmer, Leuthold, Soetens, Schröter, \& Sommer, 2002; Valle-Inclán, Hackley, \& de Labra, 2002). In terms of the alternative-routes model, this effect was originally explained as the blocking (Stoffels, Van der Molen, \& Keuss, 1989) or gating (Mordkoff, 1998) of the unconditional route by control processes. Stürmer et al. (2002), for example, suggested that the automatic response priming route can be suppressed by an ancillary monitoring mechanism (see also Leuthold \& Schröter, 2006) when there is a preceding incongruent trial. Alternatively, sequential changes in the SE have been explained as consequences of feature integration processes, which operate independently of the SE (Hommel, Proctor, \& Vu, 2004). The suggestion entails that combinations of stimulus features or stimulus-response (S-R) features are bound together into event files during the execution of a trial and that disruption of the bindings in a subsequent trial leads to a slowing of responses. Hommel et al. demonstrated that these repetition effects may mimic the sequential congruency modulation effect (see also Notebaert et al., 2001). The feature integration theory has been challenged, however, on the basis of analyses controlling for stimulus and response repetitions, showing that sequential modulations in the Simon task were not eliminated (Ullsperger, Bylsma, \& Botvinick, 2005).

Both the blocking hypothesis and the feature integration hypothesis make predictions about the influence of a preceding trial on the current trial but make no claims about what happens with these influences in the long run or how these mechanisms may be influenced by practice. In an early version of the dual-route model, Van Duren and Sanders (1988) suggested that frequent incompatible stimuli may lead to the complete blocking of the automatic route, because participants may choose "the worst case scenario." Applied to the present blocked design, one could argue that after sufficient incompatible practice, the automatic priming route is permanently blocked, leading to the absence of the SE or even a reversed effect. However, such a blocking would also lead to the absence of SEs after congruent trials, which is not what happens. 
Also, on the basis of the feature integration hypothesis, predictions can be made about the combined effects of practice and sequence. Colzato, Raffone, and Hommel (2006) found evidence that practice has no direct influence on the strength of feature binding or on the speed with which features are bound. However, they suggested that practice can have an effect on which features are considered for binding. Because, in the blocked condition, participants practice several blocks with location-relevant trials, attention may be directed toward location, so that location-response bindings may be preferred. Because binding effects are, by definition, temporal and linked to specific events (Stoet \& Hommel, 1999), they cannot explain long-term effects. According to the feature integration hypothesis, long-term modulations of the SE caused by practicing a location-relevant task should be independent of short-term sequential changes. Moreover, since practice is assumed to have no influence on the strength of binding, we would expect no change in the sequential effects over blocks. Therefore, the binding hypothesis does not predict a difference between the blocked and the mixed conditions concerning the influence of the preceding trial.

Support for the independence of both modulatory effects comes from a recent study by Iani et al. (2009). They investigated the relationship between practice-induced and sequential modulation of the SE. The authors concluded that both effects are additive and independent, with sequential modulations caused by influences on the unconditioned route, whereas practice-induced modulations were ascribed to new S-R links created in the framework of the conditional route. However, this conclusion can be challenged on the basis of their own data and analyses. In both experiments in this study, a significant three-way interaction was found between the SE, preceding congruency, and the type of practice, suggesting that sequential modulations are influenced by location-relevant practice. Especially striking in their results is the reversal of the SE after incongruent trials for the compatible group in Experiment 2, which is in contrast to the absence of a reversal in the control condition in Experiment 1. An increase of the Simon reversal effect after incongruent trials, together with an unchanging or sometimes increasing SE after congruent trials, is a strong indication for an interaction. Running ahead of the results of the present study, similar findings will be reported here systematically.

In this study, we compared the patterns of SEs in mixed and blocked conditions by using the same stimuli and timing in both conditions. We also looked at the stability of the Simon reversal effect in a delayed Simon task, after mixed conditions of location-relevant and location-irrelevant trials. The nature of the memory links was investigated by looking at the persistence of modulated SEs as a function of the preceding congruency.

As in previous studies, we expected that SEs would be modulated in both the blocked and the mixed designs. However, we predicted that the pattern would return to the level of a normal Simon task in the mixed condition, whereas in the blocked condition we expected a more permanent change in the pattern when participants had practiced with a location-relevant incompatible mapping. We assumed that this relative permanent change would be caused by the development of an LTM link between nodes representing contralateral stimulus and response locations. According to the predictions of the feature integration hypothesis, no interaction between binding and the overall size of the SE should be expected, because they are assumed to be independent processes (Hommel et al., 2004). Following the feature integration or the STM link hypothesis, sequential changes caused by the congruency of the preceding trial should only be temporary in both blocked and mixed conditions. More particularly, in the blocked, as well as in the mixed, condition, changes in the pattern of sequential effects caused by location-relevant practice should return to the level of a control condition shortly after practice. On the contrary, hypothesizing the development of a contralateral LTM link, permanent changes should be expected in the blocked condition only.

\section{EXPERIMENT 1}

In this experiment, we studied the reversal of the SE in blocked conditions. Some of the participants first performed in a location-relevant two-choice task with compatible or incompatible mapping, whereas the participants in the control condition executed a Simon task. All the participants then ran through eight blocks of a regular Simon task.

We predicted that the participants practicing with an incompatible mapping would show a decreased (or reversed) $\mathrm{SE}$, as compared with the control condition, throughout all Simon blocks, due to the creation of a contralateral LTM link. We predicted no long-term increase of the SE with compatible practice, because ipsilateral LTM links already existed before training. Importantly, the influence of binding was assessed by looking at the changes in sequential modulations of the SE and their interaction with practice condition.

\section{Method}

Participants. There were 54 participants in the experiment, divided into three groups of 18 , depending on the nature of the practice trials. Eighteen participants first executed a two-choice compatible task, 18 did an incompatible task, and 18 ran through a control condition consisting of a two-choice Simon task. The data of 4 participants in the control group were corrupted due to computer problems and could not be used for the analyses. All the participants were first-year university students, ranging in age from 18 to 25 years. They all had normal or corrected-to-normal vision and showed no color blindness.

Apparatus and program. The participants were tested individually in semidarkened cubicles in the psychological laboratory of the University of Brussels. The experiment was run on Pentium 4 personal computers with 17-in. screens, using E-Prime Version 1.1 Service Pack 3 software (Schneider, Eschman, \& Zuccolotto, 2002a, $2002 \mathrm{~b}$ ). The stimulus was a blue, red, or green circle, with a diameter of $1 \mathrm{~cm}$, appearing on a white background, $2.65 \mathrm{~cm}$ to the left or right of a central fixation cross. The locations where the stimulus could appear were indicated by two squares with sides of $2.3 \mathrm{~cm}$. At a viewing distance of $60 \mathrm{~cm}$, the between-stimulus visual angle amounted to $3.5^{\circ}$. The participants responded by pressing either the "c" or the " $n$ " key on the bottom row of a standard keyboard with the left or the right index finger, respectively. 
Table 1

Mean Reaction Times (RTs, in Milliseconds) and Error Percentages (ERs) in the Simon Task of Experiment 1 As a Function of Preceding Training Blocks and Spatial Congruency

\begin{tabular}{|c|c|c|c|c|c|c|}
\hline \multirow{2}{*}{$\begin{array}{c}\text { Training } \\
\text { Condition }\end{array}$} & \multicolumn{2}{|c|}{ Training } & \multicolumn{2}{|c|}{ Congruent } & \multicolumn{2}{|c|}{ Incongruent } \\
\hline & RT & ER & RT & $\overline{E R}$ & RT & ER \\
\hline Compatible & 286.9 & 1.11 & 403.7 & 2.93 & 434.8 & 7.55 \\
\hline Incompatible & 330.4 & 3.65 & 387.4 & 5.40 & 399.9 & 6.73 \\
\hline $\begin{array}{c}\text { Simon (control) } \\
\text { Congruent } \\
\text { Incongruent }\end{array}$ & $\begin{array}{l}376.5 \\
411.1\end{array}$ & $\begin{array}{r}3.68 \\
11.90\end{array}$ & 388.5 & 3.00 & 418.2 & 6.75 \\
\hline
\end{tabular}

Procedure. All the participants first received a training session, where they executed four blocks of 100 trials. One group, which from here on we will call the compatible group, had to react compatibly to blue stimuli appearing left or right of fixation. The second group, the incompatible group, had to react with the key on the opposite side of the location of the blue stimulus. Thus, for these two groups, stimulus location was the relevant dimension in the training session. Finally, the control group had to perform a Simon task for training. They had to react to the color of the stimulus, which was either red or green and could also appear left or right of fixation. Following the training session, all the participants ran through eight blocks of 100 trials in a Simon task. The mapping of colors onto keys was counterbalanced across participants in all groups. The time between switching the key and the appearance of the next stimulus (the response-stimulus interval [RSI]) was fixed at 1,000 msec. The sequence of stimulus presentations was randomized for all stimulus attributes, and all the stimuli had an equal chance of being presented.

RT was measured from stimulus onset to the switching of the response key. The switching terminated stimulus exposure and started the RSI. The participants were instructed to react as quickly as possible without making too many errors. During a 30 -sec rest period between series, they were informed about their error rate in the previous series.

\section{Results and Discussion}

All trials with RTs shorter than $200 \mathrm{msec}$ or longer than $2,000 \mathrm{msec}$ were considered outliers and were excluded from the analysis ( $<1 \%$ of all responses). Median RTs per condition per participant were calculated. Average RTs and error percentages (ERs) over participants are shown in Table 1 as a function of preceding practice and correspondence. Also, the data from the practice blocks are listed. Besides the data in the table, RTs were calculated for each of the experimental blocks and, also, as a function of the congruency of the preceding trial. Figure 1 shows median RTs for congruent and incongruent trials as a function of preceding congruency for the three conditions (panels A, B, and C). Figure 2 illustrates the overall SEs over blocks (panel A) and SEs after congruent (panel B) and incongruent (panel C) trials.

Practice blocks. For the control group, congruent trials $(M=387 \mathrm{msec})$ were significantly faster than incongruent trials $(M=416 \mathrm{msec})\left[F(1,13)=31.60, M S_{\mathrm{e}}=12,243\right.$, $p<.001]$. Also, the error percentages followed a similar pattern. More errors were made in the incongruent $(M=$ $11.5 \%)$ than in the congruent $(M=5.0 \%)$ condition. For the location-relevant trials, RTs were shorter than in the Simon task, and compatible reactions were faster $(M=$ $285 \mathrm{msec})$ and more accurate $(M=1.1 \%)$ than incompatible reactions $(M=330 \mathrm{msec}, 3.7 \%)$. Although we have to be careful to compare these averages because of the between-subjects design, the average RTs in the compatible and incompatible conditions were comparable to those in other serial RT studies with an RSI of 1,000 msec (e.g., Soetens, Boer, \& Hueting, 1985). It is an indication that the groups were more or less matched for general RT speed.

Simon blocks. A general repeated measures ANOVA was conducted on the RTs for the Simon blocks, with practice condition as the between-subjects factor (3 levels) and congruency of the current trial (two), blocks (eight), and preceding congruency (two) as the withinsubjects factors. To avoid confusion with the factor preceding congruency, we will talk about the $S E$ (Simon effect) factor instead of congruency of the current trial. The ANOVA showed a clear overall SE $[F(1,47)=185.4$, $\left.M S_{\mathrm{e}}=234,766, p<.001\right]$, interacting with practice condition $\left[F(2,47)=12.09, M S_{\mathrm{e}}=15,311, p<.001\right]$. SEs were comparable in the control $(M=29.7 \mathrm{msec})$ and compatible $(M=31.1 \mathrm{msec})$ conditions, but smaller in the incompatible condition ( $M=12.3 \mathrm{msec}$ ). Nevertheless, the SE was still significant in the incompatible condition $\left[F(1,47)=17.29, M S_{\mathrm{e}}=21,892, p<.001\right]$, which is remarkable because, in most other studies, sizable reversals of the SE have been reported (e.g., Tagliabue et al., 2000; Vu \& Proctor, 2008; but see Iani et al., 2009).

Congruency of the preceding trial had no main effect on RT $(F<1)$ but clearly interacted with the SE $[F(1,47)=$ 366.6, $\left.M S_{\mathrm{e}}=742,725, p<.001\right]$. There was an SE when the preceding trial was congruent $(M=67.8 \mathrm{msec})$ and a reversed SE when the preceding trial was incongruent $(M=-19.0 \mathrm{msec})$. This interactive pattern changed, however, under influence of the preceding practice session $\left[F(2,47)=4.07, M S_{\mathrm{e}}=8,236, p=.023\right]$. These interactions are clearly illustrated in the three panels of Figure 1. In the control condition, we replicated the so-called blocking of the automatic route (Stürmer et al., 2002), with a significant $\mathrm{SE}$ after congruent stimuli $[F(1,47)=$ 123.6, $\left.M S_{\mathrm{e}}=228,353, p<.001\right]$, but no SE after incongruent stimuli $\left[F(1,47)=0.76, M S_{\mathrm{e}}=1,098, p=.39\right.$; see Figure 1, panel C]. For the incompatible condition, there was an SE after a congruent trial $[F(1,47)=150.6$, $\left.M S_{\mathrm{e}}=278,196, p<.001\right]$, and a significant reversed $\mathrm{SE}$ after an incongruent trial $\left[F(1,47)=70.06, M S_{\mathrm{e}}=\right.$ $101,250, p<.001$; Figure 1, panel B]. Not self-evident was the finding of a similar pattern in the compatible condition. After congruent trials, we found a larger than normal SE $\left[M=77.4 \mathrm{msec} ; F(1,47)=233.2, M S_{\mathrm{e}}=\right.$ $430,824, p<.001]$, and after incongruent trials, the SE also reversed $\left[M=-15.1 \mathrm{msec} ; F(1,47)=11.30, M S_{\mathrm{e}}=\right.$ $16,335, p=.0015$; Figure 1, panel A; see also Iani et al., 2009]. So, although the overall SE in the compatible condition did not differ from the SE in the control condition, there seems to have been a difference in the underlying causes, as shown by the influence of the preceding trial. We will come back to this issue when we analyze the evolution of congruency effects over blocks.

A similar ANOVA was conducted on the errors. The overall pattern of results was comparable to that for the RTs, although not all differences reached the level of significance. There was an overall SE $[F(1,47)=28.74$, $\left.M S_{\mathrm{e}}=4,126, p<.001\right]$, and the interaction with practice 


\section{A}

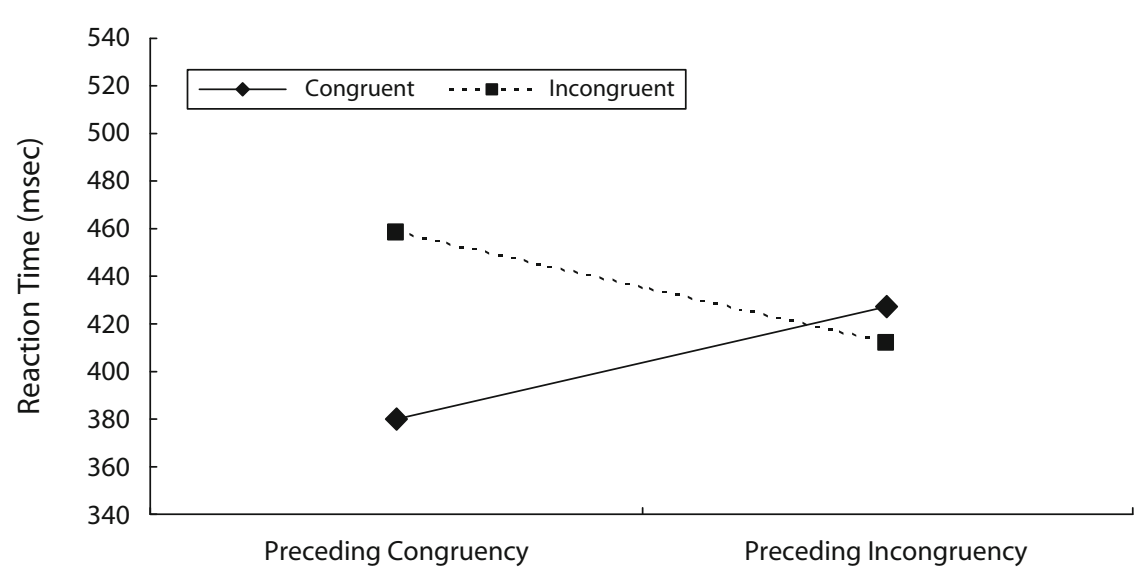

Preceding Congruency

\section{B}

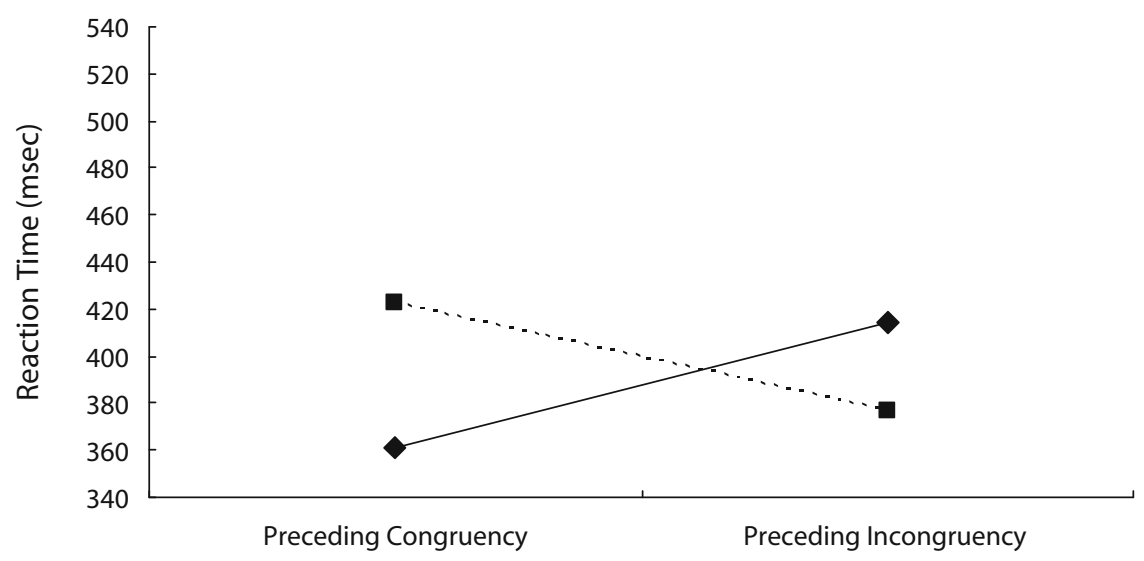

Preceding Congruency

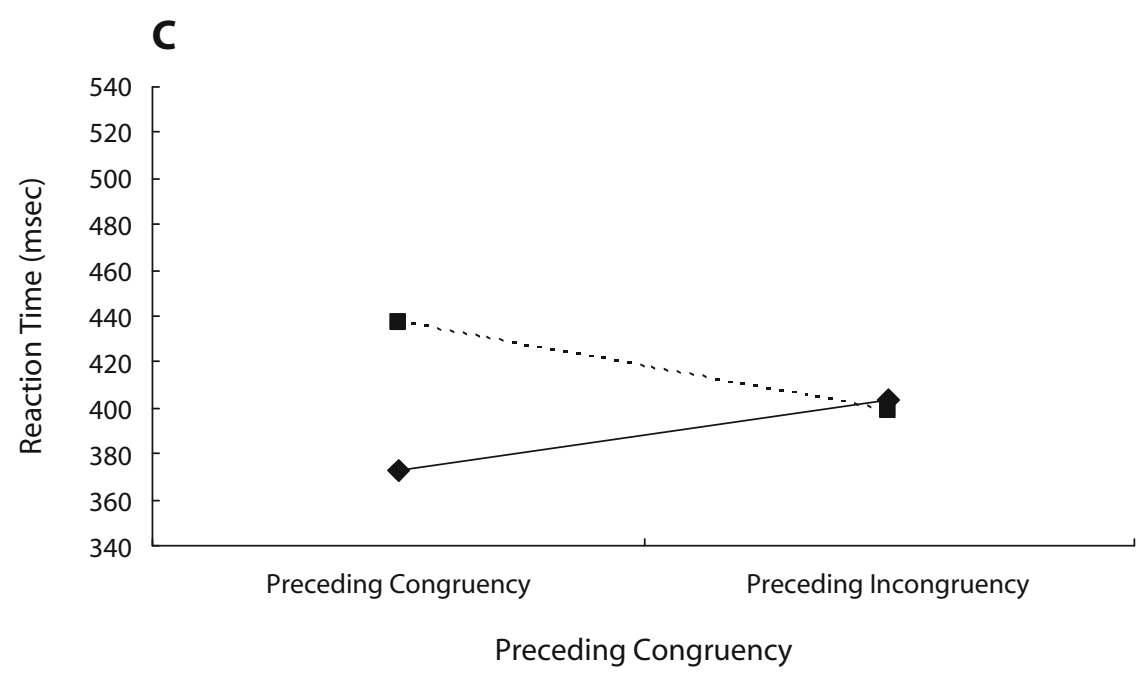

Figure 1. Experiment 1: Reaction times for congruent and incongruent trials as a function of preceding congruency for three groups of participants. (A) Data for participants with previous practice on a spatially compatible location-relevant task. (B) Data for participants with practice on a spatially incompatible task. (C) Data for participants with practice on a location-irrelevant (Simon) task. 
A

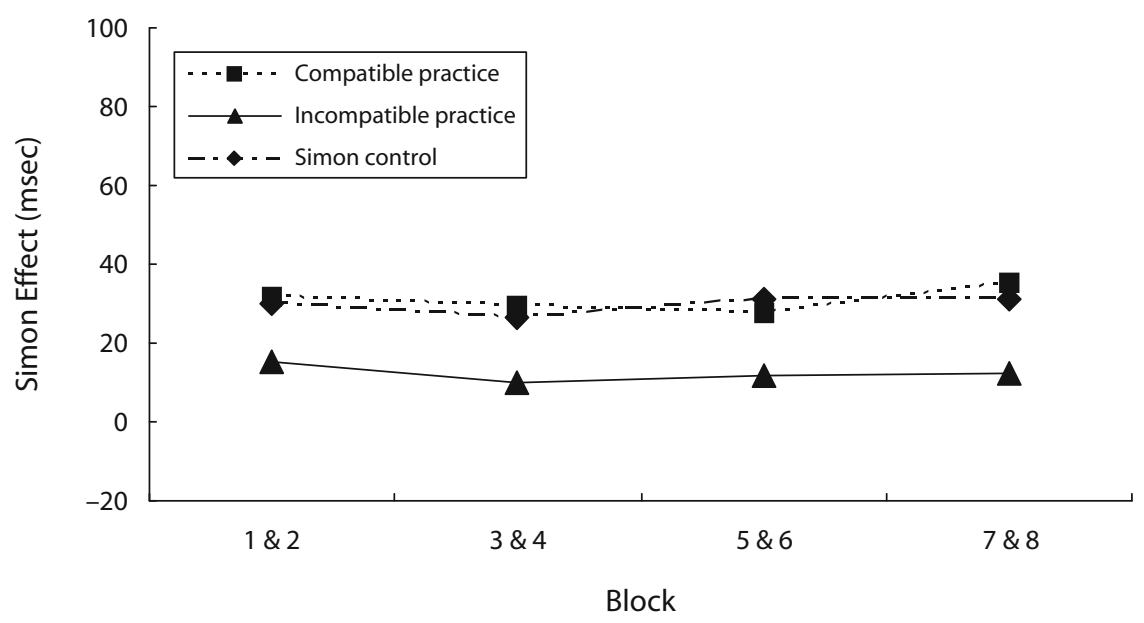

B

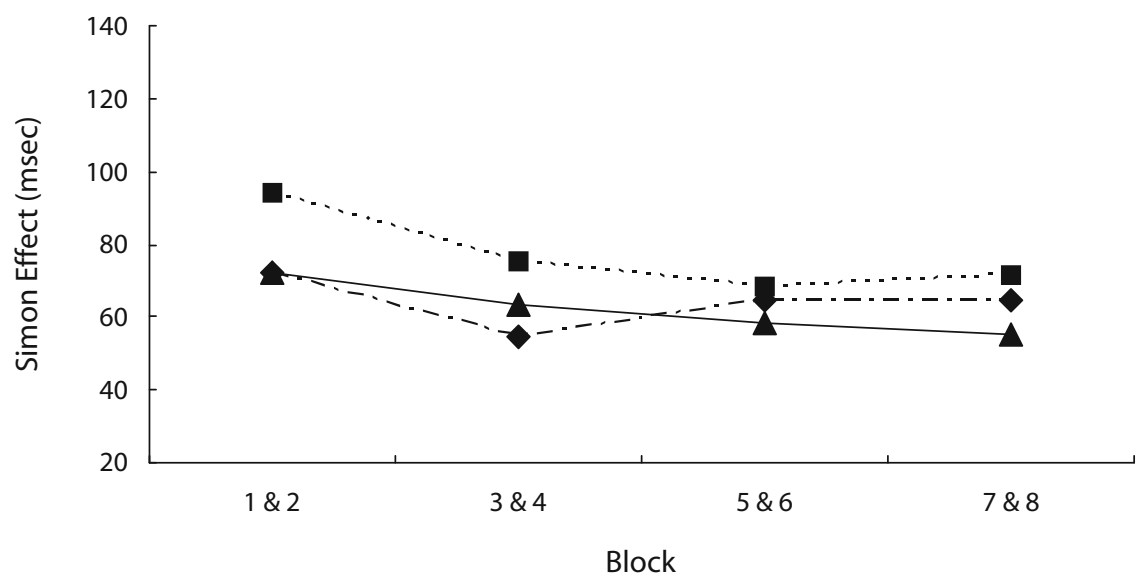

C

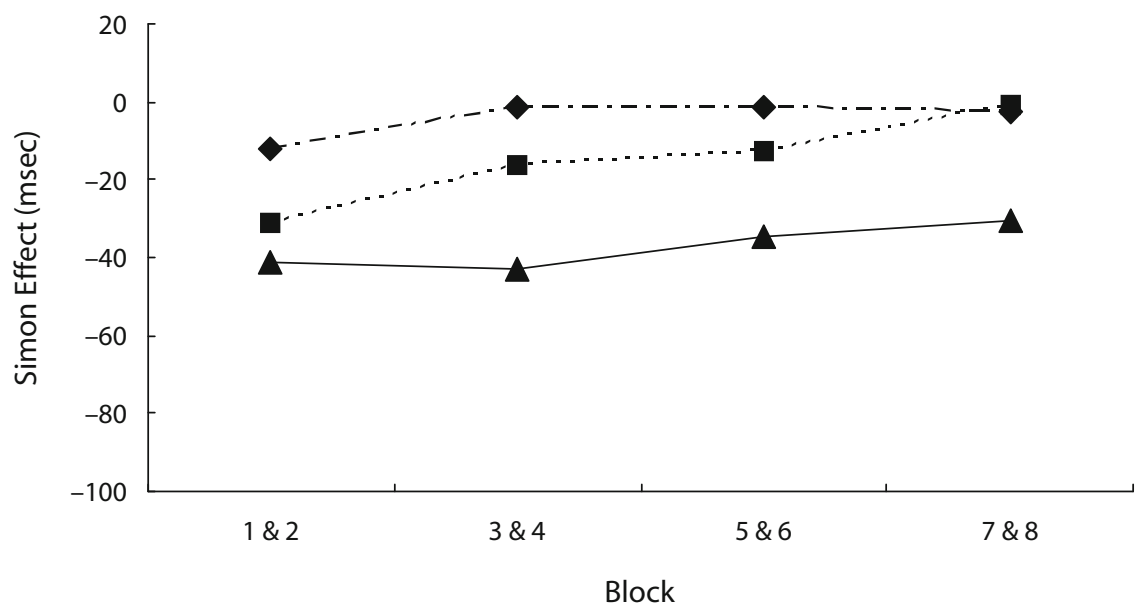

Figure 2. Simon effects (SEs; incongruent reaction time [RT] - congruent RT) over the Simon blocks in Experiment 1 for three groups of participants. The compatible group had previous practice with a spatially compatible location-relevant task, the incompatible group with a spatially incompatible location-relevant task, and the control group with a location-irrelevant (Simon) task. (A) Overall SEs. (B) SEs after congruent trials. (C) SEs after incongruent trials. 
condition was marginally significant $[F(2,47)=2.88$, $\left.M S_{\mathrm{e}}=413.69, p=.066\right]$. Again, SEs were comparable in the control $(M=3.8 \%)$ and compatible $(M=4.6 \%)$ conditions, but clearly different in the incompatible condition $(M=1.3 \%)$. In the latter case, the SE was not significant $(p=.19)$.

Also with errors, congruency of the preceding trial interacted with the SE $\left[F(1,47)=366.6, M S_{\mathrm{e}}=742,725\right.$, $p<.001]$, but this pattern did not change over practice conditions $\left[F(2,47)=1.72, M S_{\mathrm{e}}=311.5, p=.19\right]$. In all conditions, there was a significant $\mathrm{SE}$ after congruent trials. After incongruent trials, the reversed SE reached significance only in the incompatible condition. The error analyses demonstrated that the pattern of results in the RT analyses cannot be accounted for by a speed-accuracy trade-off.

Congruency over blocks. We predicted that the modulation of the SE as a consequence of incompatible location-relevant practice would be relatively permanent over blocks because of the consolidation of a contralateral LTM link. We further tested the hypothesis that sequential modulations of the SE would be only temporal and would not vary across blocks.

Figure 2, panel A, illustrates the course of SEs over blocks. In the figure, the 100-trial blocks are grouped in four blocks of 200 trials for the sake of clarity. RTs did not change over the Simon blocks $[F(7,329)=1.29$, $\left.M S_{\mathrm{e}}=3,806, p=.25\right]$ and also displayed no interaction with practice condition $\left[F(14,329)=0.90, M S_{\mathrm{e}}=2,660\right.$, $p=.56]$. Importantly, the SE did not change over blocks $\left[F(7,329)=1.13, M S_{\mathrm{e}}=663, p=.35\right]$, and this was true for all the practice conditions $\left[F(14,329)=0.90, M S_{\mathrm{e}}=\right.$ $531, p=.56]$. However, the interaction between preceding congruency and the SE did change over blocks $[F(7,329)=$ $\left.4.24, M S_{\mathrm{e}}=2,201, p<.001\right]$, and this changing pattern differed between practice conditions $[F(14,329)=1.78$, $\left.M S_{\mathrm{e}}=924, p=.040\right]$.

This higher order interaction is clearly illustrated in Figure 2, where panel B shows congruency effects over blocks after congruent trials and panel $\mathrm{C}$ after incongruent trials. After congruent trials ( panel B), there was no difference in the course of the SEs between the control and the incompatible conditions. With compatible practice, the SE was larger than in the control condition in the first two blocks $\left[F(1,47)=5.55, M S_{\mathrm{e}}=3,879, p=.023\right]$, but this difference disappeared in the last two blocks $[F(1,47)=$ $\left.0.67, M S_{\mathrm{e}}=396.7, p=.42\right]$. Interestingly, a mirrored pattern can be seen in panel C, after incongruent trials, where there was a significant increase of the reversed SE in the first two blocks after a compatible session, as compared with the control condition $\left[F(1,47)=4.15, M S_{\mathrm{e}}=\right.$ $2,901, p=.047]$, and the difference also disappeared in the last two blocks $\left[F(1,47)=0.039, M S_{\mathrm{e}}=33.4, p=\right.$ .84]. This pattern, which was limited to the condition with compatible training, is thus dual sided and results in the overall absence of a difference in SEs, as compared with the control condition. The dual-sided pattern must have been caused by prior location-relevant training, because no such effects appeared in the Simon training blocks of the control group. There, the SE was $71 \mathrm{msec}$ after con- gruent trials and $-11 \mathrm{msec}$ after incongruent trials, which is approximately the same as in the first blocks after practice for the same group. On the other hand, they were significantly different from the positive SE after congruent trials in the first two blocks of the compatible condition [92 msec; $t(30)=2.50, p=.02]$ and the reversed SE after incongruent trials $[-31.1 \mathrm{msec} ; t(30)=3.59, p<$ $.01]$. These temporary changes under the influence of the congruency sequence can be associated with binding or STM processes. The effects are clear immediately after location-relevant practice but disappear when location is no longer relevant.

A different picture emerges after incongruent trials in the incompatible condition. In panel $\mathrm{C}$, we see that, after an incongruent trial, the difference in the reversed SE between the control and incompatible conditions is large and remains stable over all blocks. The reversed effect was significant in both the first blocks $\left[F(1,47)=9.85, M S_{\mathrm{e}}=\right.$ $6,898, p=.003]$ and the last blocks $[F(1,47)=7.31$, $\left.M S_{\mathrm{e}}=6,240, p=.009\right]$, and this difference did not change over blocks $\left[F(1,47)=0.02, M S_{\mathrm{e}}=8.26, p=.89\right]$. The reversed SE after incongruent trials is the main factor in the overall decrease of the SE after incompatible training. It is clear that temporary binding processes cannot explain this more permanent sequential effect. The error analysis over blocks showed no main effect of blocks $(F<1)$ and no interaction with any of the other variables.

The analyses of the evolution of the SE as a function of congruency of the preceding trials seem to indicate that different processes are responsible for the reversal of the SE. Under influence of practice with a location-relevant compatible task, temporary changes occurred in the congruency effects, which were dual sided. Especially the increased reversal after an incongruent trial with compatible practice is striking and is in contradiction with any model that proposes the independence between practice-induced and sequential modulations of the SE. The effect of this type of practice could have been caused by STM links or bindings created during practice with location-relevant stimuli and seemed to fade away when this type of practice was stopped. Under influence of incompatible training, there was a one-sided change in congruency effects that occurred only after incongruent trials. The reversal of the SE was larger than that after incongruent trials in the compatible condition and did not decrease over blocks. This asymmetric effect may point to the creation of a contralateral LTM link.

Repetition effects. To assess the influence of feature bindings as an explanation for changes in the SE, an ANOVA was conducted with response and location sequence as within-subjects factors, instead of preceding congruency. The ANOVA included practice condition as a between-subjects factor and four within-subjects factors: block (eight levels), response sequence (two), location sequence (two), and the SE (two). Response repetitions $(M=387 \mathrm{msec})$ were faster than response alternations $(M=428 \mathrm{msec})$, whereas location sequence did not matter. Both factors did not interact with practice condition. Binding can be derived, because there was a clear interaction between response and location sequence $[F(1,47)=$ 
367.3, $\left.M S_{\mathrm{e}}=1,398,601, p<.001\right]$, and this effect was modulated by practice $\left[F(2,47)=6.25, M S_{\mathrm{e}}=23,783\right.$, $p=.004]$. Nevertheless, the interaction between response and location sequence remained significant in all three practice conditions. The binding interaction changed over blocks $\left[F(7,329)=4.07, M S_{\mathrm{e}}=3,517, p<.001\right]$, but this change was again the same for all the practice conditions $(p=.42)$. None of the interactions with the SE were significant. More particularly, the interaction between response and location sequences was unrelated to the size of the SE. From this analysis, we can conclude that there are indications that binding is influenced by the type and the amount of practice but that it does not influence the overall SE. Problematic for the feature integration hypothesis is the asymmetric sequential change in the SE after incompatible training. Whereas a strong reversal of the SE is observed after an incongruent trial, no such shift in the size of the positive SE is found after a congruent trial.

\section{EXPERIMENT 2}

In Experiment 1, we found both temporary changes of the SE and more durable effects. We suggested that the persisting reversal of the SE with incompatible practice may have been caused by a consolidation process in LTM. Such a consolidation process seems plausible because, while practicing the location-relevant incompatible task, participants have only one task set or mapping active in STM. Repeatedly using the same mapping may have created the opportunity to strengthen memory traces, leading to an LTM link.

SE reversals have also been found in experiments in which location-relevant and location-irrelevant stimuli were mixed (e.g., Proctor et al., 2000). The reversal in such experiments could be less permanent, because participants have to keep two different task sets active in STM. Consolidation of the incompatible links by location-relevant trials may be hindered by congruent trials in the locationirrelevant task.

In the present experiment, we again compared three groups of participants. One group performed in a mixed compatible condition, a second group performed in a mixed incompatible condition, and a third group executed a Simon task. The three groups were matched on their ini- tial SE. After performing in the mixed blocks, there was a delay, after which all the participants again performed a Simon task to check the persistence of the modulated congruency effects. The delay was introduced to give the participants a rest period, because the experiment was rather long (1,400 trials).

\section{Method}

Participants. Forty-six participants, divided into three groups, depending on between-subjects conditions, executed the experiment. Sixteen participants performed a mixed compatible task, 15 performed a mixed incompatible task, and 15 were in the control condition. All the participants were first-year university students, ranging in age from 18 to 25 years. They all had normal or correctedto-normal vision and showed no color blindness.

Procedure. Except for the details mentioned in this section, the experimental design and stimuli were the same as those in Experiment 1 . All the participants first ran through two matching blocks of 100 Simon trials. Three matched groups were composed on the basis of the size of the overall average RT and SE. Subsequently, there were eight blocks of 100 trials with mixed blocks, which were different for the three groups. One group reacted in a mixed compatible design, where participants had to react to stimuli of three different colors. When the stimulus was red or green, they had to respond left or right to the color of the stimulus. In case of a blue stimulus, the participants had to make a location-compatible reaction. A second group performed in a mixed incompatible design, with the participants now reacting incompatibly to the location of the blue stimuli. Finally, there was a control group with only red and green stimuli - that is, no mixed blocks. In all three conditions, colors were approximately equiprobable. For the mixed blocks this means that $33 \%$ of the trials were location relevant. The colors appeared in a pseudorandom order.

Following the mixed blocks, there was a delay of $15 \mathrm{~min}$, after which all the participants again performed a Simon task during four blocks of 100 trials. The mapping of colors onto keys was counterbalanced as much as possible across participants in all the groups.

\section{Results and Discussion}

All trials with RTs less than $200 \mathrm{msec}$ or longer than $2,000 \mathrm{msec}$ were excluded from the analysis $(<1 \%$ of all responses). Median RTs per condition per participant were calculated. After checking the quality of the matching procedure in the practice blocks, we first analyzed RTs and errors in the mixed blocks and control condition. For clarity's sake, we limited the analysis to task repeat trials - that is, Simon trials that were preceded by another Simon trial. In a second step, we analyzed the Simon delay

Table 2

Mean Reaction Times (RTs, in Milliseconds) and Error Percentages (ERs) in the Mixed Simon Task of Experiment 2, As a Function of Mixing Condition and Congruency for Matching, Mixed, and Simon Delayed Blocks

\begin{tabular}{|c|c|c|c|c|c|c|c|}
\hline \multirow[b]{2}{*}{ Mixing Condition } & \multirow[b]{2}{*}{ Congruency } & \multicolumn{2}{|c|}{ Matching } & \multicolumn{2}{|c|}{$\begin{array}{l}\text { Mixed } \\
\text { Blocks }\end{array}$} & \multicolumn{2}{|c|}{$\begin{array}{l}\text { Simon } \\
\text { Delay }\end{array}$} \\
\hline & & RT & ER & RT & ER & RT & ER \\
\hline \multirow[t]{3}{*}{ Mixed compatible } & Congruent & 371 & 5.49 & 470 & 1.30 & 375 & 4.14 \\
\hline & Incongruent & 398 & 10.27 & 504 & 6.61 & 401 & 8.37 \\
\hline & Location relevant & & & 565 & 12.40 & & \\
\hline \multirow[t]{3}{*}{ Mixed incompatible } & Congruent & 365 & 3.33 & 446 & 8.55 & 362 & 6.20 \\
\hline & Incongruent & 396 & 9.36 & 451 & 4.61 & 380 & 7.79 \\
\hline & Location relevant & & & 542 & 11.70 & & \\
\hline \multirow[t]{2}{*}{ Simon control } & Congruent & 372 & 5.01 & 379 & 3.65 & 361 & 5.23 \\
\hline & Incongruent & 402 & 11.39 & 403 & 6.17 & 387 & 8.60 \\
\hline
\end{tabular}

Note-For the mixed blocks, location-relevant data are also included. 


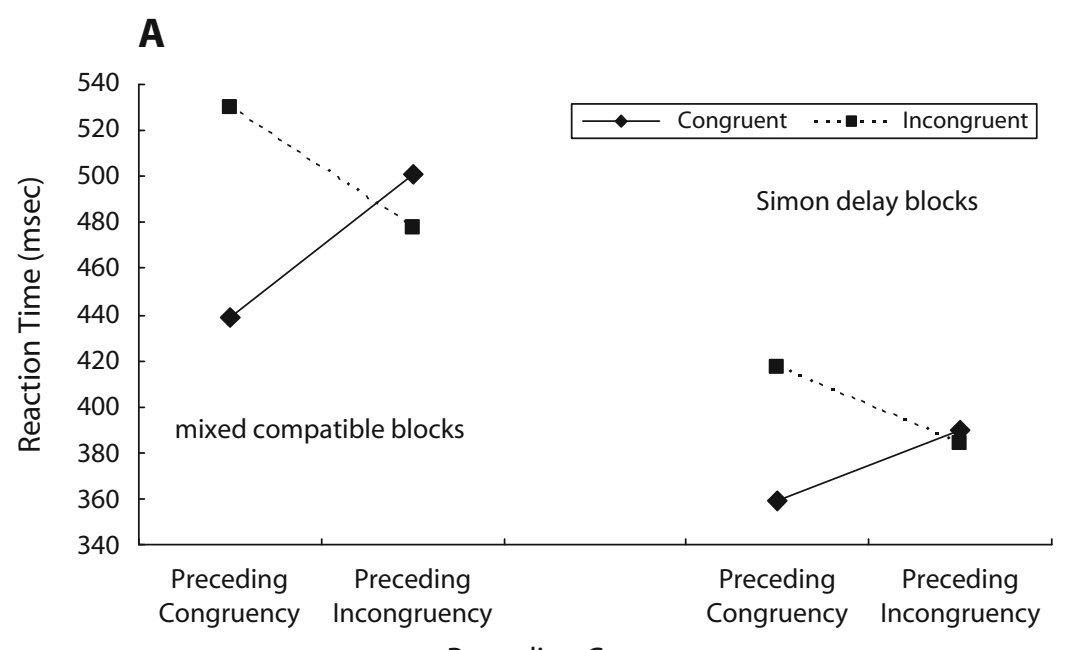

Preceding Congruency

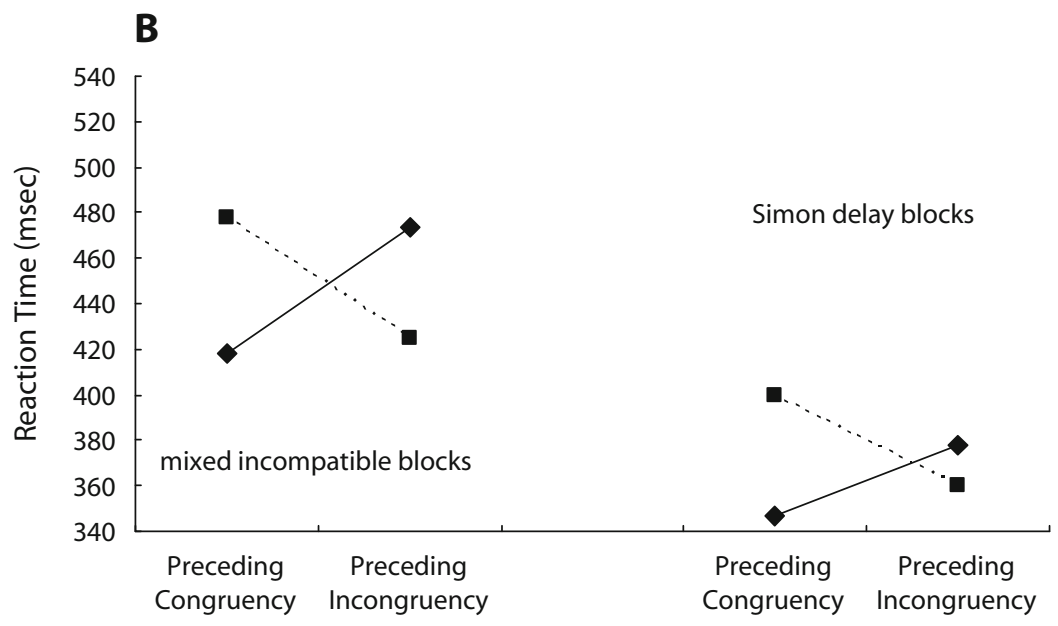

Preceding Congruency

C

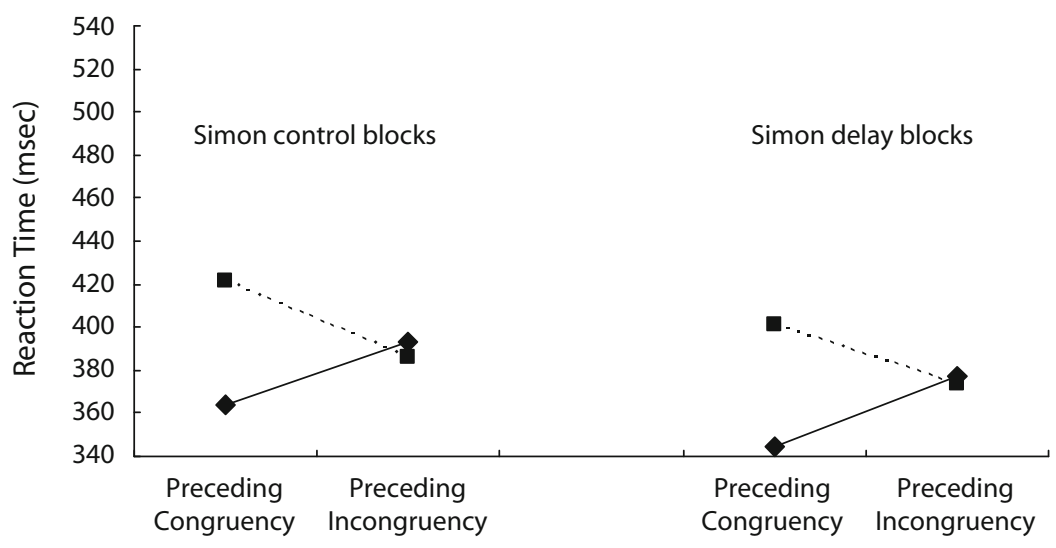

Preceding Congruency

Figure 3. Experiment 2: Reaction times for congruent and incongruent trials for mixed blocks (left) and Simon delay blocks (right) as a function of preceding congruency for three groups of participants. (A) Data for participants with mixed blocks of spatially compatible location-relevant trials and Simon trials. (B) Data for participants with mixed blocks of spatially incompatible location-relevant trials and Simon trials. (C) Data for participants in the control condition with unmixed Simon trials. 
trials to assess the stability of the congruency changes over time. RTs and errors as a function of mixing condition and congruency are shown in Table 2. Figure 3 shows RTs for congruent and incongruent trials as a function of preceding congruency for the three conditions (panels A, B, and C) for the mixed and Simon delay blocks. Finally, Figure 4 illustrates the overall SEs over blocks (panel A), and SEs after congruent (panel B) and incongruent (panel C) trials.

Matching blocks. An ANOVA was conducted with mixing condition as a between-subjects factor (three levels) and SE (two) and preceding congruency (two) as within-subjects factors. RTs did not differ between groups, and the data showed an overall SE $[F(1,43)=$ $\left.160.97, M S_{\mathrm{e}}=38,648, p<.001\right]$. Both within-subjects factors did not interact with mixing condition $(p=.74$ and .30, respectively). SEs were $26.5,30.2$, and $30.2 \mathrm{msec}$ in the mixed compatible, mixed incompatible, and control conditions, respectively. The only other significant effect was an interaction between the SE and preceding congruency $\left[F(1,43)=246.65, M S_{\mathrm{e}}=93,547, p<.001\right]$. The error analysis had an identical pattern, with a significant SE $\left[F(1,43)=38.11, M S_{\mathrm{e}}=1,508.6, p<.001\right]$ and an interaction between Simon and preceding congruency $\left[F(1,43)=116.98, M S_{\mathrm{e}}=2,745.1, p<.001\right]$. In general, we can say that the three groups were equivalent in overall RTs and showed an equally strong SE in the matching blocks.

Mixed blocks. An ANOVA was conducted on the RTs for the task repeat trials only, with mixing condition as a between-subjects factor (three levels), and blocks (eight), SE (two), and preceding congruency (two) as withinsubjects factors. The SE was clearly significant $[F(1,43)=$ 42.06, $\left.M S_{\mathrm{e}}=164,425, p<.001\right]$ and interacted with mixing condition $\left[F(2,43)=6.88, M S_{\mathrm{e}}=27,061, p<\right.$ $.01]$. An SE emerged in both the mixed compatible and control conditions ( $p<.001$ in both conditions) but was absent in the incompatible condition $(p=.39)$. The size of the SE was not different between mixed compatible and control conditions $\left[F(1,43)=1.34, M S_{\mathrm{e}}=5,252.3\right.$, $p=.25]$. Between incompatible and control, the difference was significant $\left[F(1,43)=6.00, M S_{\mathrm{e}}=23,593, p<\right.$ $.02]$. It is remarkable that, also here, the SE did not reverse in the incompatible condition, which is in contrast to most other similar studies (Marble \& Proctor, 2000; Vu \& Proctor, 2008).

In line with earlier studies and the analysis of the matching blocks, there was an interaction between the SE and preceding congruency $\left[F(1,43)=212.92, M S_{\mathrm{e}}=842,960\right.$, $p<.001]$. This interaction changed with mixing condition $\left[F(2,43)=6.03, M S_{\mathrm{e}}=23,877, p<.01\right]$, following approximately the same pattern as in the Simon blocks in Experiment 1. For the mixed blocks, these interactions are illustrated in the left panels of Figure 3. In the control condition, we again observed the blocking effect, with a significant SE after a congruent trial $(p<.001)$ and no SE after an incongruent trial ( $p=.32$; Figure 3, panel C, left figure). In mixed conditions, there was an SE after congruent trials ( $p<.001$ in both conditions) and a significant re- versed SE after incongruent trials $(p<.002$ and $p<.001$, in the mixed compatible and incompatible conditions, respectively; Figure 3, panels A and B, left figures).

Comparing the size of the SE in the mixed conditions with that in the control condition as a function of preceding congruency, we see that in the compatible condition, the positive $\mathrm{SE}$ increased when the preceding trial was congruent ( $p<.01$; see Figure 4, panel B). With a preceding incongruent trial, there was only a marginal difference with the control condition $(p=.10)$. In the mixed incompatible condition, things were the other way around. There was no difference in the size of the SE, as compared with the control, when the preceding trial was congruent $(p=.81)$, but there was a significant difference when the preceding trial was incongruent $(p<.001$; see Figure 4 , panel C).

The modulation of the SE in the mixed conditions was comparable to that found in earlier studies (e.g., Marble \& Proctor, 2000). The SE did not increase significantly under the influence of mixing location-relevant compatible trials, but the SE disappeared when Simon trials were mixed with location-relevant incompatible trials. Moreover, the pattern of congruency effects in relation to preceding congruency was comparable to that in the first blocks after practice in Experiment 1. Except for an interaction with preceding congruency $(p=.03)$, no changes were observed over the mixed blocks. More specifically, there was no four-way interaction [mixing condition $X$ blocks $\times \mathrm{SE} \times$ preceding congruency; $F(14,301)=1.40$, $p=.15$ ], showing that the pattern of SEs in all three conditions remained stable over the mixed blocks.

The error analysis mainly supported the RT findings. The main effect of congruency was marginally significant $\left[F(1,43)=3.51, M S_{\mathrm{e}}=616.35, p=.068\right]$ and showed a strong modulation with mixing condition $[F(2,43)=$ $\left.15.73, M S_{\mathrm{e}}=2,762.4, p<.001\right]$. In the mixed compatible and control conditions, the SE was positive $(p<.001$ and .05 , respectively), whereas in the mixed incompatible condition, it was negative $(p<.01)$. Also, the results of the analyses with preceding congruency followed the same pattern as that in the RT analysis. The block factor was not significant and did not interact with any other factor.

An ANOVA on the location-relevant trials in the mixed blocks, with mixing condition as a between-subjects factor (two levels), did not show an overall compatibility effect $\left[F(1,29)=0.53, M S_{\mathrm{e}}=64,684, p=.47\right]$.

Simon delay blocks. An ANOVA was performed on the RTs in the Simon delay blocks, with mixing condition (three levels) as a between-subjects factor and delay blocks (four), SE (two), and preceding congruency (two) as within-subjects factors. The SE was significant and did not interact with mixing condition. In all three conditions, there was a positive $\mathrm{SE}\left[F(1,43)=45.21, M S_{\mathrm{e}}=42,230\right.$, $p<.001 ; F(1,43)=21.61, M S_{\mathrm{e}}=20,185, p<.001 ;$ and $F(1,43)=46.73, M S_{\mathrm{e}}=43,646, p<.001$, for the mixed compatible, incompatible, and control conditions, respectively].

Figure 3, panels A, B, and C, right figures, display the interaction between the SE and preceding congruency for 


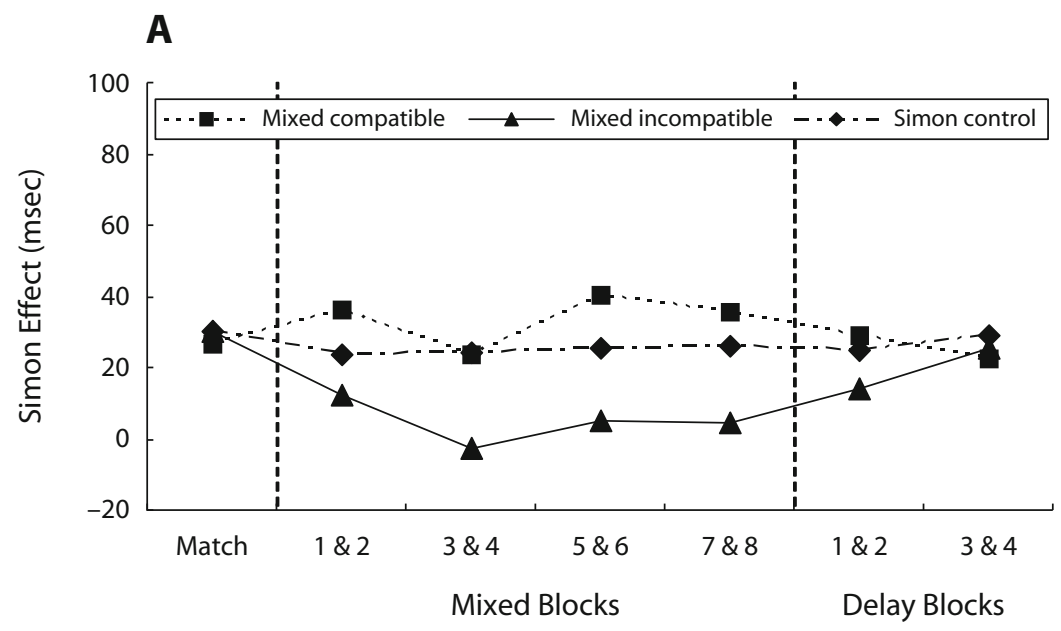

B

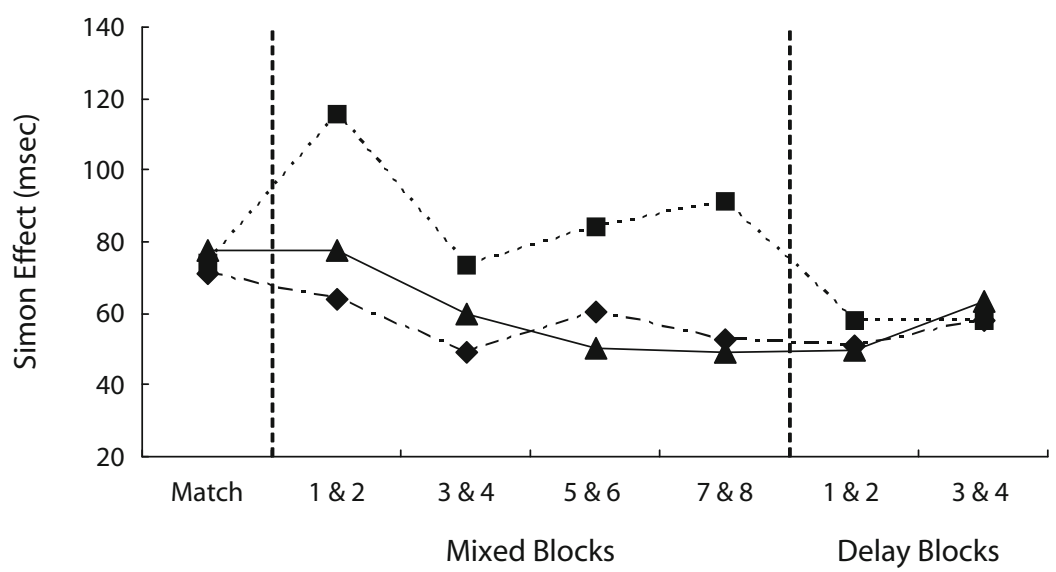

C

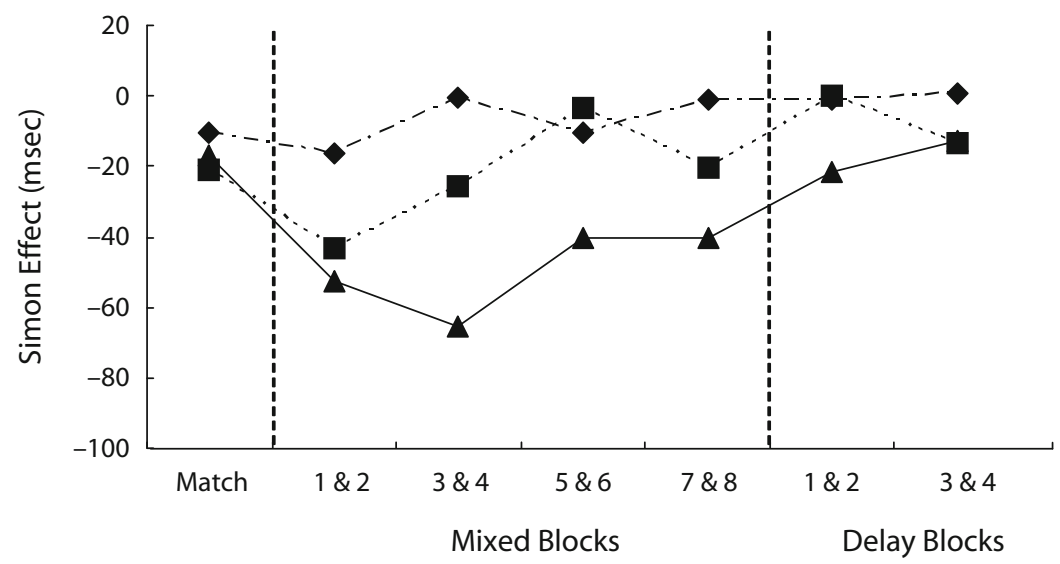

Figure 4. Simon effects (SEs; incongruent reaction time [RT] - congruent RT) over matching, mixed, and Simon delay blocks in Experiment 2 for three groups of participants. In the mixed compatible group, spatially compatible location-relevant trials were mixed with Simon trials; in the mixed incompatible group, spatially incompatible trials were mixed with Simon trials; and in the Simon control group, only location-irrelevant trials were presented. (A) Overall SEs. (B) SEs after congruent trials. (C) SEs after incongruent trials. The SEs in the mixed blocks show only the data for task repeat trials. 
the three conditions. As could be expected, the interaction was significant $\left[F(1,43)=180.3, M S_{\mathrm{e}}=179,511\right.$, $p<.001]$, and did not differ between mixing conditions $[F(2,43)=0.73, p=.49]$. Panel B in Figure 4 shows that RTs in the Simon delayed blocks in the three conditions overlapped after a congruent trial. In panel C, however, we see that a reversed SE lingered on after an incongruent trial in the mixed incompatible condition but decayed slowly. However, there was no significant four-way interaction [mixing condition $\times$ blocks $\times \mathrm{SE} \times$ preceding congruency; $F(6,129)=0.60, p=.72]$. This may have been caused by the gradual decrease of the reversed SE in the Simon delay blocks and because the effect had already diminished substantially in the first delay block. Comparing the reversal effect for the mixed incompatible group between the mixed and the Simon delay blocks shows a clear interaction $[F(1,43)=22.76, p<.001]$, confirming that the reversal strongly decreased in the Simon delay blocks $(M=-16.0 \mathrm{msec})$, as compared with the mixed blocks $(M=-49.7 \mathrm{msec})$. In contrast to the other two conditions, where there was no SE after an incongruent trial, the reversed SE in the mixed incompatible condition was still significant in Simon Delay Blocks 1 and 2 $\left[F(1,43)=8.98, M S_{\mathrm{e}}=6,605, p<.01\right]$, but not any more in Delay Blocks 3 and $4\left[F(1,43)=3.22, M S_{\mathrm{e}}=940.8\right.$, $p=.08]$. The difference in the reversal of the SE after an incongruent trial between the mixed compatible and control conditions, which was $39.1 \mathrm{msec}$ in the last mixed blocks, diminished to $11.2 \mathrm{msec}$ in the last Simon delay blocks.

The analysis of the errors in the delay blocks confirmed the RT results. There was a significant overall SE $\left[F(1,43)=19.23, M S_{\mathrm{e}}=433.5, p<.001\right]$, interacting with preceding congruency $\left[F(1,43)=85.3, M S_{\mathrm{e}}=\right.$ $1,732, p<.001]$. This pattern was not influenced by mixing conditions.

In general, the data showed that the changes in the SE caused by the mixing conditions were temporary and that the RT pattern returned to a normal SE, including the reversed effect after incongruent trials, when no more location-relevant trials were mixed. This can be considered to be an indication that the mixing of location-relevant and location-irrelevant trials only modulated STM links.

Repetition effects. Also, the data in Experiment 2 were analyzed for repetition effects with an ANOVA similar to that in Experiment 1. We confined the analysis to the Simon delay blocks, because we were interested mainly in the interaction between the persistence of SE and binding processes, as expressed in the response $X$ location sequence interaction. Response, as well as location, repetitions were faster than alternations $[F(1,43)=$ 224.0, $M S_{\mathrm{e}}=822,569, p<.001$, and $F(1,43)=6.34$, $M S_{\mathrm{e}}=7,452, p=.01$, respectively]. They showed a mutual interaction $\left[F(1,43)=149.2, M S_{\mathrm{e}}=294,653, p<\right.$ .001], again indicating feature binding, but this was not different across mixing conditions $(F<1)$. Moreover, this interaction did not enter into any higher order interaction with the SE. In contrast to Experiment 1, binding does not seem to be influenced by mixing condition, but in agreement with Experiment 1, binding does not influence the SE.

\section{GENERAL DISCUSSION}

In two experiments, we compared the modulation of the SE caused by location-relevant reactions in blocked and mixed designs. In Experiment 1, practicing with a spatially compatible task had no influence on the overall size of the SE in a subsequent location-irrelevant task. Practicing with a spatially incompatible task, however, led to a strong decrease of the SE. The modulation of the SE persisted and did not decrease during the execution of eight blocks of 100 trials on a location-irrelevant task. In Experiment 2, where location-relevant and location-irrelevant trials were mixed, a similar asymmetrical pattern emerged. Mixing spatially compatible trials with Simon trials did not influence the overall size of the SE, whereas the effect was eliminated when spatially incompatible trials were mixed. However, in contrast to Experiment 1, the modulation of the SE disappeared rapidly during four delayed Simon blocks. The pattern of SEs shows that changes in memory links occurring in the blocked design are different from those occurring in the mixed designs. The differentiation between the conditions was further clarified by analyzing sequential modulations of the $\mathrm{SE}$.

In the following, we will first discuss the results in terms of STM and LTM links. Subsequently, we will look at the sequential modulations of the SE under the influence of compatible and incompatible location-relevant tasks or trials. We will demonstrate that extra assumptions are needed to accommodate the existing models to the present data. Finally, we will suggest an alternative explanation for the observed patterns of SEs.

\section{Short- and Long-Term Memory Links}

In the blocked design of Experiment 1, we replicated the modulation of the SE under the influence of practicing with a location-relevant spatial compatibility task prior to executing a Simon task. Practicing with a compatible mapping did not alter the size of the SE, which is similar to the results found by Tagliabue et al. (2000; see also Proctor $\& \mathrm{Lu}, 1999)$. Our data therefore support the suggestion of these authors that LTM links that cause the regular SE have been overlearned and, therefore, their strength cannot be further increased. With incompatible practice, the effect was strongly reduced, and even reversals of the SE have been observed (Proctor \& Lu, 1999; Tagliabue et al., 2000; Vu \& Proctor, 2008). Importantly, we found that the reduction after incompatible practice persisted over 800 location-irrelevant trials. Also this result is in accordance with other studies (Tagliabue et al., 2002; Tagliabue et al., 2000; Vu et al., 2003), where reductions of the effect have been found from $24 \mathrm{~h}$ to 1 week after practice.

A remarkable difference between the present data and those in most other, similar studies is the absence of a reversed SE after incompatible practice or with mixing Simon and incompatible trials. (e.g., Marble \& Proctor, 2000; Proctor \& Lu, 1999; Tagliabue et al., 2000; Vu, 
2007; Vu et al., 2003). Likely, the difference is related to the size of the SE (on average, $35 \mathrm{msec}$ in the present study, as compared with $16 \mathrm{msec}$ in, e.g., Vu et al., 2003), and this, in turn, may have been caused by stimulus eccentricity $\left(3.5^{\circ}\right.$ in the present study, vs. $7.5^{\circ}$ and more in most other studies). Hommel (1993) demonstrated the importance of stimulus eccentricity for the size of the SE and even showed a small but insignificant reversal when stimuli were highly eccentric. Other support for an explanation in terms of stimulus eccentricity has come from the study of Iani et al. (2009), who also did not find a reversal of the SE, although the participants had an incompatible training of 384 trials. In their study, the stimuli were presented with an eccentricity of $5^{\circ}$, which is close to that in the present study.

In the mixed design of Experiment 2, we also found a strong decrease of the SE when location-relevant trials were incompatible, whereas no change was found with a compatible mixture. Again, in contrast to earlier studies with mixture designs, there was no reversal of the overall SE. Again, these differences may be explained by the influence of stimulus eccentricity. Proctor and colleagues (Marble \& Proctor, 2000; Proctor et al., 2000; Proctor \& $\mathrm{Vu}, 2002)$ also found that the SE in their mixed compatible condition was significantly larger than that in the control condition. However, the percentage of location-relevant trials $(50 \%)$ was higher in these studies than in the present Experiment 2 (33\%). The effects of relative frequency of corresponding trials are presumed to have been due to attentional biases, favoring the more frequent type of trials (Logan, 1980; Logan \& Zbrodoff, 1979). These effects have been associated with changes in STM links.

In contrast to the blocked design, the decreased SE in the mixed incompatible condition returned to the size in the control condition in the course of four blocks of 100 Simon trials. This shows that the changes occurring in the $\mathrm{SE}$ as a consequence of mixing location-relevant and location-irrelevant trials were only temporary. On the basis of the comparison between the results of the two experiments, we argue that the changes that occurred in the SE in the mixed experiment were a consequence of adaptations of STM links, whereas the changes that occurred in the blocked experiment were caused by the consolidation of an LTM link.

Tagliabue et al. (2000) argued that the formation of a new contralateral LTM link is implausible because it cannot explain why there is a shift of the reversed SE to longer RTs, as compared with the regular SE. They suggested that the reversal effect is caused by long-lasting STM links, instead of LTM links, and that the extra hidden layer of STM nodes slows down the contralateral link, resulting in stronger SEs with longer RTs. The model of Tagliabue et al. (2000) is an important improvement, as compared with the older model of Zorzi and Umiltà (1995), because it makes a clear distinction between STM and LTM links, and also because it introduces a difference in the temporal aspects of these links. To explain the reversal of the SE, the authors considered two possible adaptations to the memory links: on the one hand, the formation of a new contralateral LTM link, and on the other hand, the development of a long- lasting contralateral STM link. They rejected the formation of a new LTM link because it failed to capture the time course of the effect, as shown by their bin analyses, and concluded that the existence of long-lasting STM nodes better explains the observed patterns of SEs. In our opinion, the model is still an oversimplification of the memory processes involved. First, we agree with the suggestion of STM links, because participants are instructed to perform a specific task and, therefore, whether the task is spatially compatible or incompatible, a task set is always present in STM and, thus, STM links should be present in both congruent and incongruent tasks. The question is why the authors suggested existence of an STM link for incompatible tasks, but not for compatible tasks. Second, the model does not provide an explanation of how an STM link might ultimately develop into an LTM link. We suggest that with incompatible practice, STM links are indeed produced for the spatial nodes. However, subsequently, these new contralateral links automize over time and develop into LTM links. So, the difference we want to stress, as compared with the model of Tagliabue et al. (2000) is that, at some point in time, a new LTM link is created, parallel to the STM link. Moreover, we think that such a new LTM link is not of the all or nothing type but that its strength develops gradually. Many studies have shown that the formation of LTM representations is a gradual process (e.g., Soetens, Casaer, D'Hooge, \& Hueting, 1995), taking much more time than the formation of an STM representation. A more plausible explanation for the failure to simulate the results on the basis of long-term links and for the asymmetry in ipsilateral and contralateral SEs has to be looked for in the differences in strength between a newly formed LTM link and an "old" LTM link. Anyhow, this explanation is more in line with the accepted definitions of STM and LTM as generally used in the memory literature. Therefore, we suggest that a new LTM link develops that is comparable to, but weaker than, the older LTM link on which the positive $\mathrm{SE}$ is built.

Why can LTM links develop in the blocked experiment and not in the mixed experiment? The explanation above seems plausible in view of the difference in task set between the two experiments (Proctor \& Vu, 2002). In the blocked condition, the participants had only one task set in STM during the spatially incompatible practice session, whereas two task sets had to be kept in memory in the mixed experiment. Moreover, because practice happens with location-relevant trials only, the memory links may have grown stronger than those developed by taskirrelevant spatial attributes. These factors may have contributed to a fast consolidation of the incompatible LTM link in the blocked experiment. In the mixed experiment, however, one task set entailed the mapping of a left stimulus to a right response and vice versa, whereas the other task set entailed the mapping of colors to keys. Because, on $50 \%$ of these trials, the participants made use of the congruent LTM links, consolidation of the incompatible links may have been hindered. In general, the increased load of STM may have hampered the consolidation process, leading to less permanent modulations of the SE in the mixed condition. 
Three alternative causes for the difference in persistence of memory traces can be pointed out. The total number of location-relevant trials was 400 in the blocked condition versus approximately 266 in the mixed condition $(33 \%$ of 800$)$. However, it is unlikely that the 134 extra trials can explain the stability of the effect over eight blocks of 100 Simon trials, whereas the modulation could not be maintained in only four Simon delay blocks after the mixed practice. A second possible explanation for the difference in persistence is that the participants performed a pure Simon task for 200 trials prior to the mixed task in Experiment 2. It is not clear, however, how these extra trials could have influenced the persistence of the reversal, because they were neutral with respect to the S-R mapping, in that the numbers of corresponding and noncorresponding trials were equal. Moreover, the incompatible condition in the mixing experiment produced a stronger decrease of the SE, as compared with the same condition in the blocked experiment, suggesting that the extra practice with Simon trials did not hamper the formation of contralateral links. A third difference between the experiments is the delay of $15 \mathrm{~min}$ introduced after the mixed condition, which may have attenuated the strength of the memory traces. However, a delay after locationrelevant practice does not seem to decrease the reversal (Vu et al., 2003) but, rather, increases the effect (Tagliabue et al., 2000).

\section{Sequential Effects}

Sequential modulations of the SE have been explained as temporary effects on the RT of the current trial by the congruency or the features of the preceding trial. According to the blocking or gating hypothesis (Mordkoff, 1998; Stürmer et al., 2002), the automatic response priming route is controlled after an incongruent trial. In both experiments, we replicated the blocking effect in the control conditions. There was an SE of approximately $60 \mathrm{msec}$ after congruent trials but no SE after incongruent trials. However, the changes in sequential effects were different between the two experiments. Whereas there were temporary changes in the sequential modulations in the mixing experiment, a mixture of temporary and more permanent changes was observed in the blocked experiment. Both the blocking and the feature integration hypothesis predict only temporary changes and make no clear predictions about long-term effects. Moreover, feature integration predicts sequential modulations independent of the overall SE and has no explanation for a difference in long-term changes in the SE after congruent and incongruent trials.

Location-relevant compatible stimuli. With locationrelevant compatible stimuli, changes occurring in the SE under the influence of the preceding trial are approximately the same in mixed and blocked experiments. They can be summarized as follows: There is a temporary increase in the SE after a congruent trial and a temporary increase of the reversal of the SE after an incongruent trial, as compared with the control group. The increased SE after a congruent trial can easily be accommodated by assuming the formation of an STM link, boosting the normal SE of the LTM link. The effect is only temporary, because after the task set is no longer in effect, the STM link is no longer of use and fades away. After eight blocks of Simon stimuli in a blocked experiment and after four Simon delay blocks in a mixed condition, the SE after a congruent trial is the same as that in the control condition. Also, the feature integration hypothesis has no difficulty in accommodating this result. With compatible practice, attention is drawn to the location feature of the stimulus, so that bindings of this feature are more likely to occur (Colzato et al., 2006), resulting in a stronger SE.

The increased reversal of the SE after an incongruent trial, however, is more difficult to reconcile with this assumption. Although the effect is only temporary, it points to the creation of a contralateral link and, thus, cannot be explained by the formation of an ipsilateral STM link under the influence of practice. Also, the feature integration hypothesis, which assumes that sequential modulation occurs independently of the overall SE (Hommel et al., 2004), cannot explain the increased reversal. Increased reversal can also be observed in the study of Iani et al. (2009), although it went unobserved because a comparison had to be made over experiments. In their control condition in Experiment 1, which is comparable to the present control condition, there was no SE and no reversal after an incongruent trial, either in the RTs or in the error rate. In their horizontal compatible condition in Experiment 2, however, there was a reversal of the SE, probably only marginally significant in the RTs, but clearly significant in the error rates. According to the feature integration hypothesis, an overall shift of the SE to more positive is to be expected after compatible training, which is exactly the opposite of what the data show after an incongruent trial. An explanation in terms of the blocking or gating mechanism may be possible, provided that we make the extra assumption that the blocking mechanism reacts more strongly when an incongruent event is exceptional (Stürmer et al., 2002). As blocking occurs more often, as in the case of a Simon task after compatible practice, the blocking mechanism returns to its neutral level, explaining the temporary character of the increased reversal. We will come back to this issue when we propose an alternative explanation for these results.

Location-relevant incompatible stimuli. In contrast to the conditions with compatible stimuli, with incompatible stimuli the pattern of sequential effects is asymmetrical, and moreover, the results between blocked and mixed experiments are not the same. In both experiments, the size of the SE after a congruent trial is the same as in the control condition. So, although the overall SE has decreased considerably with incompatible practice or stimuli, there is no influence at all after a congruent trial. On the other hand, there is a large increase in the reversal of the SE after an incongruent trial. Importantly, the increase of the reversal is temporary in the mixed condition and relatively permanent in the blocked condition.

As was explained above, the asymmetric pattern caused by incompatible practice can be explained by the formation of a contralateral STM or LTM link. The contralateral LTM link explains the more permanent character of the Simon reversal after incongruent trials with incompatible 
practice (see also Tagliabue et al., 2002; Tagliabue et al., 2000). Again, the shift of the SE after incongruent trials only argues against the feature integration account, because it clearly predicts an overall shift of the SE to more negative, independently of the congruency of the preceding trial. Moreover, since both the blocking and the feature integration accounts predict only temporary effects, they have no explanation for why the reversal is more permanent in the blocked, as compared with the mixed, condition. A possible solution could be that, in contrast to earlier claims (Colzato et al., 2006), practice does influence the speed of the formation of bindings.

Although it is clear from the preceding that changes in the SE under the influence of location-relevant practice are not independent of changes in sequential modulations, the extra ANOVAs with feature repetition effects did not show any interaction between binding (response $X$ stimulus location interaction) and the SE. Indirectly, this could be interpreted as support for the idea that binding is not responsible for the sequential changes observed in these experiments. Although more research is necessary to strengthen this claim, it adds to the earlier counterarguments (e.g., Ullsperger et al., 2005).

\section{A Bottom-Up Priming Mechanism}

The data from the present study, and also from other studies, suggest that, in contrast to earlier claims (e.g., Iani et al., 2009), the two factors causing the modulation of the SE are not independent and that an explanation is needed for their interaction. In the following, we will make an alternative suggestion about how these mechanisms interact, and we will then take a look at how the present and earlier findings are in agreement with this proposition.

The basic assumption we make is that preceding events activate or prime existing memory links, a claim that is quite common in the memory literature (e.g., repetition priming; Berry, Shanks, \& Henson, 2008). If we accept that, in a Simon task, different links can be created between stimulus- and response-related nodes, as has been suggested by several authors (Proctor, Vu, \& Marble, 2003; Tagliabue et al., 2000; Zorzi \& Umiltà, 1995), a selection mechanism is needed to explain why a specific link is preferred on a specific trial. The default option is that all available links are used equally, but it is clear from earlier modeling efforts that this cannot explain the modulations of the SE. The suggested priming or preactivation mechanism creates a direct link between bottom-up and top-down mechanisms.

The suggestion for such a selection process in congruency tasks is not new. The blocking or gating mechanism is of a similar nature, in that the use of an LTM link is conditional upon the congruency of the preceding trial (Stoffels et al., 1989; Stürmer et al., 2002). Also, the attention shift hypothesis (Notebaert \& Soetens, 2003; Notebaert et al., 2001; Stoffer \& Yakin, 1994), suggested as an explanation for the regular SE, has been presented as a kind of priming mechanism. Shifting attention spatially in a particular direction is assumed to prime responses located in the same direction. The suggestion of memory priming that we propose here is more general, applying to all memory links, and is not limited to one unconditional route. It is a parsimonious explanation, because no blocking of an automatic route is needed.

Priming can explain why there is an SE after a congruent trial, and not after an incongruent trial. A preceding congruent trial activates the LTM route, leading to the $\mathrm{SE}$ on the following trial. A preceding incongruent trial primes a task-relevant STM link, which will be used on the following trial. Priming is not a blocking mechanism, inhibiting all nonprimed routes. If preceding events are neutral with respect to the irrelevant spatial dimension, there will still be a small advantage for the nonprimed LTM link, resulting in a decreased SE after neutral trials (Wühr \& Ansorge, 2005).

We now return to the formation of memory links caused by the present manipulations and explain how priming can cause the differential influence of preceding congruent and incongruent trials on the SE in different practice conditions.

\section{Priming and Practice}

The persistent decrease of the SE after incompatible practice in the blocked experiment can be ascribed completely to an increased reversed SE after incongruent trials. No change was observed after congruent trials. According to the priming hypothesis, the newly formed contralateral LTM link is activated only after an incongruent trial, leading to a persisting reversed effect. After a congruent trial, participants barely make use of this new LTM link, because it has not been primed on the preceding trial.

Alternatively, blocking of the new LTM link could be suggested here, but a general priming mechanism is more parsimonious, because it can also explain why there is a normal-sized SE after a congruent trial. It shows that now, the "old" LTM route is preferred after priming, leading to fast congruent trials and slow incongruent trials. The fact that the positive SE after congruent trials is larger than the reversed effect after incongruent trials can be explained by a difference in strength of the LTM links. It is evident that links that have been trained over many years are stronger than those developed in merely 400 location-relevant incompatible trials. No other mechanisms of blocking, gating, or binding are needed. Tagliabue et al. (2000) found the same results for an adult population but a reversed SE for children, larger than the positive SE in a mixed compatible condition. These authors speculated that the ipsilateral LTM links developed in children are not yet strong enough and are, therefore, more affected by compatibility training.

In the blocked compatible condition, only STM links were influenced, because the ipsilateral LTM link is assumed to be there already. A preceding congruent trial could have activated both STM and LTM links, leading to a temporary increase of the SE in the first blocks after practice. It is not clear why there was also an enhanced reversal effect, as compared with the control condition, after an incongruent trial, because no strong incongruent STM links could have been developed with locationrelevant compatible practice. It cannot be explained by the influence of practice in general, because no such increase 
of the reversal was observed in the Simon training blocks for the control group in Experiment 1. Possibly, training with location-relevant trials in general attracts attention to the spatial dimension, so that it temporarily increases the congruency effects. Such an attentional mechanism may also explain why Iani et al. (2009, Experiment 2) found a reversal of the left-right SE following an incongruent trial after training with a vertical compatible task, as compared with no reversal in a control condition without prior practice. Although the vertical setup can be considered neutral as to the left-right dimension, here, attention was also drawn to the spatial feature of the stimuli

Most of the explanations for the blocked experiment also apply to the mixed experiment. In the incompatible condition, the overall decrease of the SE, as compared with the control condition, was again caused by an increased reversal after incongruent trials. However, the effect did not persist but returned to the level of the control condition in subsequent delayed Simon trials. This suggests that only contralateral STM links were affected by the mixing, because participants did not have the opportunity to consolidate the link into LTM. Because the reversal was conditional upon the preceding incongruence, we assume that the preceding trial primed the STM link, leading to an enhanced reversal.

In the mixed compatible condition, the pattern was very similar to that in the blocked experiment. The SE increases temporarily in the first blocks after congruent trials, and the reversed SE increases temporarily after incongruent trials. The reversal was smaller than that in the mixed incompatible condition. This difference may have been caused by the frequency of incompatible mappings, because there were more incompatible reactions in the mixed incompatible condition. In both cases, an incongruent trial primed the STM link, leading to an enhanced reversal effect. After a congruent trial, the participants could make use of the "old" LTM link, leading to a positive SE, comparable to that in the control condition. In contrast to Marble and Proctor (2000; Proctor et al., 2003), we suggest that the "old" LTM link, which is responsible for the SE proper, was still active in mixed conditions. The authors above based their conclusion on the fact that the Simon reversal effect in the mixed incompatible condition was at least as large as the positive SE in the mixed compatible condition. This was not the case in the present study, where we found an overall decrease of the SE only in the mixed incompatible condition. The difference between their results and ours cannot be explained by a frequency effect ( $50 \%$ location-relevant trials in Marble \& Proctor [2000], as compared with only $33 \%$ in the present setup), because a strong reversal was also found by $\mathrm{Vu}$ and Proctor (2008), where 33\% location relevant trials also were used. As was explained before, stimulus eccentricity may be the factor responsible for this difference.

Our main argument, however, for suggesting that the old LTM link remains active is based on the influence of preceding congruency. After a congruent trial, in both the mixed compatible and the mixed incompatible conditions, the SE was at least as large as in the pure Simon blocks $(70-80 \mathrm{msec})$. If the LTM route were not active in the mixed conditions, we would have expected a smaller positive SE, as compared with the control condition. A second argument that has been used by Marble and Proctor (2000), which is also applicable to the present study, is the absence of a compatibility effect in the location-relevant trials of the mixed blocks. However, it is difficult to interpret this between-subjects difference, because, in general, participants reacted more quickly $(23 \mathrm{msec})$ in the mixed incompatible condition than in the compatible condition.

\section{Conclusions}

The studies on the reversal of the SE have shown that the explanation of congruency effects on the basis of a dual-route model have to be revisited. Explanations in terms of STM and LTM links in different stages of development are an important improvement in this respect. In this study, we found that the pattern of modulations of the SE in blocked and mixed designs can be explained by the interaction of STM and LTM links. The asymmetrical development of the modulations in both types of designs can, to a large part, be explained by a strong LTM link between location-compatible stimulus and response nodes that have been developed over a lifetime. The persistent reversal of the SE in the blocked designs could have been caused by the development of a new LTM link for incompatible S-R relations, whereas the temporal reversal of the effect in the mixed design represented modulations in STM links. To explain the interaction between the influence of the congruency of the immediately preceding trial and the effects over longer intervals, we suggest that STM and LTM links are primed by preceding events, making them more receptive for subsequent processing.

\section{AUTHOR NOTE}

Correspondence concerning this article should be addressed to E. Soetens, Department of Cognitive and Biological Psychology, University of Brussels, Pleinlaan 2, B-1050 Brussels, Belgium (e-mail: esoetens@vub.ac.be).

\section{REFERENCES}

BARBER, P., \& O'LeAry, M. (1997). The relevance of salience: Towards an activation account of irrelevant stimulus-response compatibility effects. In B. Hommel \& W. Prinz (Eds.), Theoretical issues in stimulusresponse compatibility (pp. 135-172). Amsterdam: North-Holland.

Berry, C. J., Shanks, D. R., \& Henson, R. N. A. (2008). A singlesystem account of the relationship between priming, recognition, and fluency. Journal of Experimental Psychology: Learning, Memory, \& Cognition, 34, 97-111.

Colzato, L. S., Raffone, A., \& Hommel, B. (2006). What do we learn from binding features? Evidence for multilevel feature integration. Journal of Experimental Psychology: Human Perception \& Performance, 32, 705-716.

Hommel, B. (1993). The relationship between stimulus processing and response selection in the Simon task: Evidence for a temporal overlap. Psychological Research, 55, 280-290.

Hommel, B., \& Prinz, W. (EDs.) (1997). Theoretical issues in stimulusresponse compatibility. Amsterdam: North-Holland.

Hommel, B., Proctor, R., \& Vu, K.-P. L. (2004). A feature-integration account of sequential effects in the Simon task. Psychological Research, 68, 1-17.

Iani, C., Rubichi, S., Gherri, E., \& Nicoletti, R. (2009). Co- 
occurrence of sequential and practice effects in the Simon task: Evidence for two independent mechanisms affecting response selection. Memory \& Cognition, 37, 358-367.

Kornblum, S., HasbroucQ, T., \& Osman, A. (1990). Dimensional overlap: Cognitive basis for stimulus-response compatibility-A model and taxonomy. Psychological Review, 97, 253-270.

Kornblum, S., \& STEvens, G. (2002). Sequential effect of dimensional overlap: Findings and issues. In W. Prinz \& B. Hommel (Eds.), Common mechanisms in perception and action: Attention and performance $X I X$ (pp. 9-54). Oxford: Oxford University Press.

Leuthold, H., \& Schröter, H. (2006). Electrophysiological evidence for response priming and conflict regulation in the auditory Simon task. Brain Research, 1097, 167-180.

LoGAN, G. D. (1980). Attention and automaticity in Stroop and priming tasks: Theory and data. Cognitive Psychology, 12, 523-553.

LoGan, G. D., \& ZBRodofF, N. J. (1979). When it helps to be misled: Facilitative effects of increasing the frequency of conflicting stimuli in a Stroop-like task. Memory \& Cognition, 7, 166-174.

Marble, J. G., \& Proctor, R. W. (2000). Mixing location-relevant and location-irrelevant choice-reaction tasks: Influences of location mapping on the Simon effect. Journal of Experimental Psychology: Human Perception \& Performance, 26, 1515-1533.

MoRdKoFf, J. T. (1998). The gating of irrelevant information in selectiveattention tasks. Abstracts of the Psychonomic Society, 3, 21.

NotebaerT, W., \& SoETEns, E. (2003). An auditory attention shift primes a corresponding response. Psychological Research, 67, 253-260.

Notebaert, W., Soetens, E., \& Melis, A. (2001). Sequential analysis of a Simon task -Evidence for an attention-shift account. Psychological Research, 65, 170-184.

Proctor, R. W., \& Lu, C.-H. (1999). Processing irrelevant location information: Practice and transfer effects in choice-reaction tasks. Memory \& Cognition, 27, 63-77.

Proctor, R. W., Marble, J. G., \& Vu, K.-P. L. (2000). Mixing incompatibly mapped location-relevant trials with location-irrelevant trials: Effects of stimulus mode on the reverse Simon effect. Psychological Research, 64, 11-24.

Proctor, R. W., \& VU, K.-P. L. (2002). Eliminating, magnifying, and reversing spatial compatibility effects with mixed location-relevant trials. In W. Prinz \& B. Hommel (Eds.), Common mechanisms in perception and action: Attention and performance XIX (pp. 443-473). Oxford: Oxford University Press.

Proctor, R. W., Vu, K.-P. L., \& Marble, J. G. (2003). Mixing locationrelevant and irrelevant tasks: Spatial compatibility effects eliminated by stimuli that share the same spatial codes. Visual Cognition, 10, 15-50.

Schneider, W., Eschman, A., \& Zuccolotto, A. (2002a). E-Prime reference guide. Pittsburgh: Psychology Software Tools.

Schneider, W., Eschman, A., \& Zuccolotto, A. (2002b). E-Prime user's guide. Pittsburgh: Psychology Software Tools.

Simon, J. R. (1990). The effects of an irrelevant directional cue on human information processing. In R. W. Proctor \& T. G. Reeve (Eds.), Stimulus-response compatibility: An integrative perspective (pp. 3186). Amsterdam: North-Holland.

Simon, J. R., \& Rudell, A. P. (1967). Auditory S-R compatibility: The effect of an irrelevant cue on information processing. Journal of Applied Psychology, 51, 300-304.

Soetens, E., Boer, L. C., \& Hueting, J. E. (1985). Expectancy or automatic facilitation? Separating sequential effects in two-choice reac- tion time. Journal of Experimental Psychology: Human Perception \& Performance, 11, 598-616.

Soetens, E., Casaer, S., D'Hooge, R., \& Hueting, J. (1995). Effect of amphetamine on long-term retention of verbal material. Psychopharmacology, 119, 155-162.

Stoet, G., \& Hommel, B. (1999). Action planning and the temporal binding of response codes. Journal of Experimental Psychology: Human Perception \& Performance, 25, 1625-1640.

Stoffels, E. J., Van der Molen, M. W., \& Keuss, P. G. J. (1989). An additive factor analysis of the effect(s) of location cues associated with auditory stimuli on stages of information processing. Acta Psychologica, 70, 161-197.

StofFer, T. H., \& YAKIN, A. R. (1994). The functional role of attention for spatial coding in the Simon effect. Psychological Research, 56, $151-162$.

Stürmer, B., Leuthold, H., Soetens, E., Schröter, H., \& SomMER, W. (2002). Control over location-based response activation in the Simon task: Behavioral and electrophysiological evidence. Journal of Experimental Psychology: Human Perception \& Performance, 28, 1345-1363.

Tagliabue, M., Zorzi, M., \& Umiltà, C. (2002). Cross-modal remapping influences the Simon effect. Memory \& Cognition, 30, 1823.

Tagliabue, M., Zorzi, M., Umiltà, C., \& Bassignani, F. (2000). The role of long-term memory and short-term-memory links in the Simon effect. Journal of Experimental Psychology: Human Perception \& Performance, 26, 648-670.

Ullsperger, M., Bylsma, L. M., \& Botvinick, M. M. (2005). The conflict adaptation effect: It's not just priming. Cognitive, Affective, \& Behavioral Neuroscience, 5, 467-472.

Valle-Inclán, F., Hackley, S. A., \& de Labra, C. (2002). Does stimulus-driven response activation underlie the Simon effect? In W. Prinz \& B. Hommel (Eds.), Common mechanisms in perception and action: Attention and performance XIX (pp. 474-493). Oxford: Oxford University Press.

Van Duren, L. L., \& SAnders, A. F. (1988). On the robustness of the additive factors stage structure in blocked and mixed choice reaction designs. Acta Psychologica, 69, 83-94.

VU, K.-P. L. (2007). Influences on the Simon effect of prior practice with spatially incompatible mappings: Transfer within and between horizontal and vertical dimensions. Memory \& Cognition, 35, 1463 1471.

VU, K.-P. L., \& Proctor, R. W. (2008). Age differences in response selection for pure and mixed stimulus-response mappings and tasks. Acta Psychologica, 129, 49-60.

Vu, K.-P. L., Proctor, R. W., \& Urcuioli, P. (2003). Transfer effects of incompatible location-relevant mappings on a subsequent visual or auditory Simon task. Memory \& Cognition, 31, 1146-1152.

WÜHR, P., \& ANSORGE, U. (2005). Exploring trial-by-trial modulations of the Simon effect. Quarterly Journal of Experimental Psychology, 58A, 705-731.

Zorzi, M., \& Umiltì, C. (1995). A computational model of the Simon effect. Psychological Research, 58, 193-205.

(Manuscript received October 19, 2009; revision accepted for publication December 17, 2009.) 